\title{
The relative pathogenicity of Candida krusei and C. albicans in the rat oral mucosa
}

\author{
Y. H. SAMARANAYAKE, P. C. WU, L. P. SAMARANAYAKE* and P. L. HO $\dagger$ \\ Departments of Pathology and $\uparrow$ Microbiology, Faculty of Medicine and ${ }^{*}$ Faculty of Dentistry, University of \\ Hong Kong, Hong Kong
}

\begin{abstract}
The relative pathogenicity of Candida krusei and $C$. albicans was investigated by assessing their colonisation and infectivity of the Sprague-Dawley rat oral mucosa. During an initial 21-week period with intermittent oral inoculation, both Candida spp. demonstrated variable surface colonisation of the oral mucosa. After 3 days of oral inoculation, both yeast species were recovered from all animals. During the 21-week period the mean oral load of $C$. albicans in the control group of rats varied between $(26-274) \times 10^{1} \mathrm{cfu} / \mathrm{ml}$ whereas the two test groups of rats carrying C. krusei CK9 and CK13 had a mean load of $(2-10) \times 10^{1} \mathrm{cfu} / \mathrm{ml}$. Although oral colonisation by $C$. albicans was greater than that of $C$. krusei, neither species induced candidal infection during this period. Subsequent immunosuppression of the rats by intramuscular cyclophosphamide $(40 \mathrm{mg} / \mathrm{kg}$ body weight) initiated $C$. albicans infection of the dorsal tongue (around the conical papillae area) after 4 weeks, in all of three animals while similar lesions due to $C$. krusei were seen - albeit after 5-7 weeks - in three of eight animals. Characteristic histological changes of mucosal candidosis were discernible on the lingual mucosa of rats infected with both Candida spp. including parakeratosis, absence of a stratum granulosum, thickened rete ridges, micro-abscess formation and polymorph infiltration of the lingual epithelium. Although both species produced fungal hyphae that penetrated the epithelium up to the prickle cell layer, $C$. albicans hyphae tended to be relatively more profuse. Taken together these results substantiate, for the first time in an animal model, the clinical evidence that $C$. krusei, once considered an innocuous commensal, is capable of transforming into an invasive pathogen under conditions of immunosuppression.
\end{abstract}

\section{Introduction}

The role played by various Candida spp. in initiating oral epithelial lesions is ill understood. Cawson and Lehner [1] first suggested a possible aetiological relationship between leukoplakia and candidal infection on finding mycelial elements of $C$. albicans infiltrating the cornified layer of the epithelium in some cases of human oral leukoplakia [2]. Since then several workers have attempted to produce lesions resembling human oral leukoplakia in cultured tissue [3], chick chorioallantoic membrane [4] and animal models [5, 6]. Jones and Russell $[7,8]$ pioneered these animal studies and demonstrated histological changes similar to the human disease in the posterior dorsum of the tongue of

Received 28 Nov. 1997; revised version accepted 4 March 1998.

Corresponding author: Professor L. P. Samaranayake.
Sprague-Dawley rats chronically infected with Candida spp. Since then, the Sprague-Dawley rat has served as one of the more useful animal models for investigating the epithelial changes caused by Candida spp. in the oral cavity [9-11]. However, the data from the latter studies almost exclusively pertain to $C$. albicans, the most virulent Candida spp., and indicate that the hostfungal interactions depend to a large extent on the nature of the yeast strain used, as well as the carbohydrate and antibiotic content of the rat diet $[9,12]$.

Despite the relative abundance of data on oral lesions induced by $C$. albicans in animal models, there is sparse information on the less pathogenic species of Candida such as $C$. krusei. As it is well established that patients with leukaemia, bone marrow transplants and human immunodeficiency virus infection may be readily infected by these lesser known emerging pathogens [13-15], it is important to establish their 
pathogenic attributes in vivo with an animal model. Therefore, the aims of the present investigation were to compare the potential of C. krusei and C. albicans to colonise and infect the oral mucosa in a conventional Sprague-Dawley rat model, and to assess the susceptibility to mucosal infection with these yeasts following immunosuppression by intravenous cyclophosphamide.

\section{Materials and methods}

\section{Candida isolates and growth conditions}

A strain of $C$. albicans (OSU \#3) that previously had produced lesions in a Sprague-Dawley rat model $[9,10]$ was kindly supplied by Dr C. M. Allen, of the Ohio State University College of Dentistry, Columbus, $\mathrm{OH}$, USA. Two different isolates of C. krusei (CK9 and CK13) were tested; both were isolated from oral lesions of denture stomatitis patients attending the Oral Medicine Clinic of the Glasgow Dental Hospital and School, Glasgow. These two isolates were specifically selected from a battery of $20 \mathrm{C}$. krusei isolates because of their greater virulence attributes of adherence and hydrophobicity as described in previous studies $[16,17]$. All three isolates were speciated by the germ-tube test and API20C identification test kits (API Laboratory Products, Basingstoke, Hants). They were stored in distilled water at $-70^{\circ} \mathrm{C}$ [18], subcultured monthly on Sabouraud's Dextrose Agar (SDA; Gibco Ltd, Paisley) and maintained at $4^{\circ} \mathrm{C}$ during the experimental period. Purity of cultures was ensured by regular identification.

\section{Sprague-Dawley rats}

A total of 15 locally bred, 4-week-old male rats $(200 \mathrm{~g}$, average weight) was used for the study. For the experiment, the animals were randomly assigned into three groups of five rats each and each group was housed in a separate metal cage in a conventional animal house. The three groups were coded as the control group - for inoculation with C. albicans - and test groups 1 and 2 for inoculation with C. krusei CK9 and CK13, respectively. The rats were fed a carbohydrate-rich diet (CRD) for 22 weeks (PMI Feeds Incorporation, USA). Thereafter they were given standard rat pellets for a further 7 weeks. All rats were given tetracycline hydrochloride $0.1 \%$ in their drinking water throughout the experimental period as described by Russell and Jones [19].

\section{Yeast inoculum}

The C. albicans and C. krusei isolates were cultured on SDA for $24 \mathrm{~h}$ at $37^{\circ} \mathrm{C}$. The harvested organisms were resuspended in sterile distilled water to give a yeast concentration of $c .10^{8}$ cells $/ \mathrm{ml}$, as assessed by haemocytometric counting (Hawksley Limited, London). Microscopic examination of gram-stained colo- nies of the two Candida spp. revealed no hyphal formation.

\section{Oral inoculation of Sprague-Dawley rats with Candida spp.}

Initially, the 15 rats were given tetracycline $0.1 \%$ in their drinking water ad libitum for a period of 4 weeks. Their oral cavities were then swabbed and examined to rule out indigenous candidal growth, before experimental inoculation with the respective Candida isolate. For the latter purpose, all rats were inoculated orally with $0.1 \mathrm{ml}$ of the yeast inoculum $\left(10^{7}\right.$ yeasts $\left./ \mathrm{ml}\right)$, with the aid of a sterile pipette, three times per week over the 29-week experimental period.

\section{Quantification of oral carriage of Candida spp.}

Following inoculation, a semi-quantitative estimation of the Candida spp. on oral tissues was performed once every week by a single operator. For this purpose, an experienced technician restrained each rat on a smooth surface, by holding the body with one hand and opening the mouth by pressing firmly on either side of the temperomandibular joint area with the thumb and fingers of the other hand. This method of opening the mouth facilitated both the inoculation of yeasts and subsequent swab sampling. In order to avoid crosscontamination of yeast strains the animals were handled with sterile cotton gloves designated for each group.

The oral cavity of each rat was sampled thoroughly with a dry sterile cotton wool swab. This was accomplished by inserting the swab into the mouth up to the oropharynx and firmly sampling the tongue and the mucosal surfaces by rotating the swab for $c .5 \mathrm{~s}$. The swab contents were then dispersed by mild agitation in $5 \mathrm{ml}$ of sterile distilled water; $50 \mu \mathrm{l}$ of the latter suspension were then spiral plated (Spiral Systems, $\mathrm{OH}, \mathrm{USA}$ ) on to SDA and incubated at $37^{\circ} \mathrm{C}$ for $48 \mathrm{~h}$ to estimate the number of cfu of candida (gram-stained smears of the suspensions examined microscopically revealed no hyphal formation). The identity of Candida isolates was confirmed once every 2 weeks to rule out exogenous oral yeast infection, if any.

\section{Initial gross observations of the oral tissues and subsequent experimental protocol}

At the end of 21 weeks of oral inoculation with the Candida strains, the oral cavities of all rats were examined under short ether anaesthesia. Each rat was held by a technician as described previously, the mouth was opened and the tongue and oral mucosa including the palate were thoroughly examined by a single examiner with a magnifying glass, overhead lamp and a bright light. No grossly visible candidal infection or 'white patches' were noted in the Sprague-Dawley rat oral mucosa during this period of inoculation with 
either $C$. albicans or $C$. krusei. To substantiate these gross findings, at the end of 21 weeks, a histopathological study of the tongues of two animals from the $C$. albicans group, and one each from the two C. krusei groups was also performed. The criterion used to select these four rats was their consistently high oral yeast load compared with the values for the other animals in their respective groups.

The tongue was examined visually, dissected and immersed in buffered formalin $10 \%$, for $48 \mathrm{~h}$. Three areas of the tongue were sampled for histopathological studies. The length of the samples, measured sagittally along the mid-dorsum comprised: (i) a 2-mm crosssectional length anterior to the conical papillae area; (ii) the length of the whole conical papillae area (c. $4 \mathrm{~mm}$ ); and (iii) a 4-mm cross-sectional length posterior to the conical papillae area. These three regions were separated by horizontal sectioning of the tongue. Any lesions observed on the separated pieces were identified and blocked; the dimensions of these blocks depended on the size of the lesion encountered. The blocks were embedded in paraffin and serial 5$\mu \mathrm{m}$ thick sections were cut from each specimen. Randomly selected tissue sections (one from every 10 serial sections) were stained with haematoxylin and eosin, mounted in permount and examined for histological changes, in a conventional manner. In addition, periodic acid-Schiff stained sections were used to visualise mycelial penetration of the lingual epithelium.

\section{Immunosuppression by cyclophosphamide}

The remaining 11 animals (three from the $C$. albicans group and four each from the $C$. krusei groups) were immunosuppressed with cyclophosphamide (EndoxanASTA; ASTA Medica AG, Germany). The rats were anaesthetised with ether, and venous blood samples were collected from the rat tail end before the initial administration of cyclophosphamide. After the blood was collected, cyclophosphamide ( $200 \mathrm{mg}$ dissolved in $10 \mathrm{ml}$ of sterile distilled water) was injected intramuscularly at a dose of $40 \mathrm{mg} / \mathrm{kg}$ body weight. Repeated doses of cyclophosphamide were administered at 1week intervals to all the remaining rats for a period of 8 weeks. On these occasions, venous blood samples were collected before cyclophosphamide administration and all blood samples were subjected to leucocyte counts to assess the degree of leucopenia. For this purpose, a 1 in 20 dilution of blood was made in acetic acid $2 \%$ to lyse red cells and the leucocytes were counted with a Neubauer counting chamber [20].

Naked eye examinations of the tongue and mucosal surfaces of the 11 remaining rats were made once weekly after blood was collected from the animals. All the animals were restrained and examined by a single examiner in a standard manner, with a magnifying glass, overhead lamp and a bright light. Whenever a 'white patch' with an associated lesion was seen on gross examination, the animal was killed and samples were subjected to histopathological examination as described above.

\section{Results}

The experiment comprised two parts. In the first part the rats were maintained in their normal healthy state albeit with a carbohydrate-rich diet (CRD) and with tetracycline in their water, while in the second part they were, in addition, immunocompromised by the administration of cyclophosphamide.

\section{Leucocyte counts}

The leucocyte counts of all the animals were depressed consistently immediately after administration of cyclophosphamide. The total mean (SD) leucocyte count at the beginning of the experiment (i.e., week 21 , before cyclophosphamide administration) for the control group inoculated with $C$. albicans was $1.40(0.38) / \mu 1 \times 10^{4}$ and then fell to $0.5(0.20) / \mu 1 \times 10^{4}, 1$ week after cyclophosphamide administration (end of week 22). During the following 4 weeks all three rats demonstrated a consistent depression in their leucocyte counts, at the end of which the mean (SD) leucocyte count was $0.31(0.23) / \mu 1 \times 10^{4}$. At this stage all three rats had white patches on their lingual surfaces.

In test group 1 (inoculated with $C$. krusei CK9), the mean (SD) leucocyte count was $1.60(0.36) / \mu 1 \times 10^{4}$ before cyclophosphamide administration and, 1 week after drug administration (at the beginning of week $22)$, the count decreased to $0.61(0.16) / \mu 1 \times 10^{4}$. Thereafter during the weeks before they were killed, all four rats in this group showed considerable fluctuation in counts, but in general a gradual overall reduction. Two animals (T1.1 and $\mathrm{T} 1.3$ ) whose leucocyte counts gradually declined during the experimental period (seventh and fifth week after cyclophosphamide administration) had white patches on their tongue surfaces. However, lesions were not observed in a third animal (T1.2) whose leucocyte counts declined dramatically $\left(0.30 / \mu 1 \times 10^{4}\right)$ at the end of the eighth week, and in another animal (T1.5) with a high leucocyte count $\left(3.10 / \mu 1 \times 10^{4}\right)$. These results demonstrate that the leucocyte counts of the rats did not stabilise, but showed considerable fluctuation during the 8 weeks of drug administration. The second test group of rats which harboured $C$. krusei CK13 on the lingual surface showed similar variations in leucocyte counts, and a relatively comparable association between the numbers of leucocytes and the development of white patches.

Thus, rats inoculated with $C$. albicans showed an invariable association between decreased leucocyte counts and the development of white patches, whereas 
this relationship was less clear in the two groups harbouring $C$. krusei.

\section{Oral carriage of Candida spp. in Sprague-Dawley rats}

Before cyclophosphamide administration. None of the Sprague-Dawley rats in the three designated groups was colonised by Candida spp. at the beginning of the experiment. At the end of week 1, after three inoculations, Candida spp. were recovered from the mouths of all animals. A marked variation in the mean oral candidal load among the three groups was observed during the experimental period (Fig. 1).

The weekly variation in the load of C. albicans in the five animals in the control group was quite similar. Thus during the first 7 weeks of the experiment, the mean candidal load varied between $(26-32) \times$ $10^{1} \mathrm{cfu} / \mathrm{ml}$, but thereafter between weeks 8 and 14 the group as a whole demonstrated a gradual increase in yeast load varying between $(37-274) \times 10^{1} \mathrm{cfu} / \mathrm{ml}$. Oral colonisation began to decline during weeks $20-$ 21 in all animals (Fig. 1a).

The C. krusei load also varied considerably during weeks 1-21 (Fig. $1 \mathrm{~b}$ and c) although the relative candidal load was consistently (3-4-fold) lower than with $C$. albicans $(\mathrm{p}<0.001)$. The $C$. krusei group as a whole demonstrated an increased mean yeast load during weeks $12-16$, after which the candidal load varied widely (Fig. 1).

A variable load of $C$. krusei was also seen within each group of rats. For instance, in the group inoculated with strain CK13, two rats demonstrated a consistently high oral load when compared with the other three counterparts throughout the experimental period (data not shown).

After cyclophosphamide administration. Generally, the mean candidal load in all three groups of rats was consistently higher before cyclophosphamide administration than in the post-administration period. Furthermore, the load of $C$. albicans was relatively (12-fold) higher than that of either $C$. krusei isolate. The results of oral carriage of yeasts in the three groups of rats after cyclophosphamide administration are summarised in Fig. 1. It should be noted that none of the weekly oral samples revealed hyphal elements of either candidal species, throughout the study period. The above data demonstrated clearly the superior ability of C. albicans to colonise the Sprague-Dawley rat oral mucosa when compared with $C$. krusei, both in immunocompetent and immunosuppressed animals.

Pathological findings before cyclophosphamide administration. At the end of 21 weeks of oral inoculation with $C$. albicans, a histopathological study of the rat tongue was conducted on two randomly selected animals. These two rats showed no evidence of candidal infection in either the haemotoxylin and eosin or periodic acid-Schiff stained sections, although the candidal load in the rats ( $\mathrm{C} 3$ and $\mathrm{C} 4)$ at the time of death varied from $22.3 \times 10^{1}$ to $30.48 \times 10^{1} \mathrm{cfu} / \mathrm{ml}$.

No grossly visible lesions were noted in the SpragueDawley rat oral mucosa during the first 21 weeks of oral inoculation with either C. krusei CK9 or CK13. Histopathological examination of a randomly selected rat from each group provided no histological evidence of candidal infection, although both rats (T1.4 and T2.2) harboured $C$. krusei during the 21-week period. When they were killed, the oral candidal load in the two rats was $2.03 \times 10^{1} \mathrm{cfu} / \mathrm{ml}$.

Pathological findings after cyclophosphamide administration. On naked eye examination of the control group inoculated with $C$. albicans, white patches on the dorsal tongue mucosa were evident, with depapillation of the conical papillae region and smooth erythematous areas anterior and posterior to this region. An increasing incidence of such lingual lesions was observed in all animals after administration of cyclophosphamide, from the second week onwards (Table 1, Fig. 2). Thus all three rats in the control group demonstrated white patches on the tongue surface during week 25 . The oral candidal load for two animals, when they were killed, were similar $\left(6.09 \times 10^{1} \mathrm{cfu} / \mathrm{ml}\right)$; the third showed a higher candidal load $\left(30.48 \times 10^{1} \mathrm{cfu} / \mathrm{ml}\right)$. The diameters of the lingual lesional areas of these rats were: $2-3 \mathrm{~mm}, 1.8-2.4 \mathrm{~mm}$ and $2.8 \mathrm{~mm}$ respectively, indicating a lack of a relationship between the oral candidal load and the size of the lesion.

Histopathological changes of tissue sections incorporating the white patches demonstrated loss of normal lingual papillae and replacement by a smooth layer of parakeratotic and acanthotic epithelium (Fig. 3a). The rete ridges appeared thickened with blunt tips. The superficial layers of epithelium showed marked intercellular and intracellular oedema with polymorph infiltration. Intra-epithelial vesicles were noted in the prickle cell layer, many containing polymorphs. The basal and parabasal cells were hyperplastic with increased mitotic figures and loss of nuclear palisading. The corium showed slightly increased vascularity and mild oedema with scanty infiltrate of mononuclear cells. Fungal hyphae were observed penetrating mainly the parakeratin layer of the superficial epithelium (Fig. 3b). The slender hyphae invaded the cornified epithelium at right angles to the epithelial surface, but terminated short of the prickle cell layer. The length of $C$. albicans hyphae varied between 5 and $17 \mu \mathrm{m}$. No Candida elements were identified in the intra-epithelial vesicles. No microscopic abnormality was identified in sections taken from the grossly normal parts of the tongue.

The animals had to be immunocompromised for a longer period with cyclophosphamide for lingual 

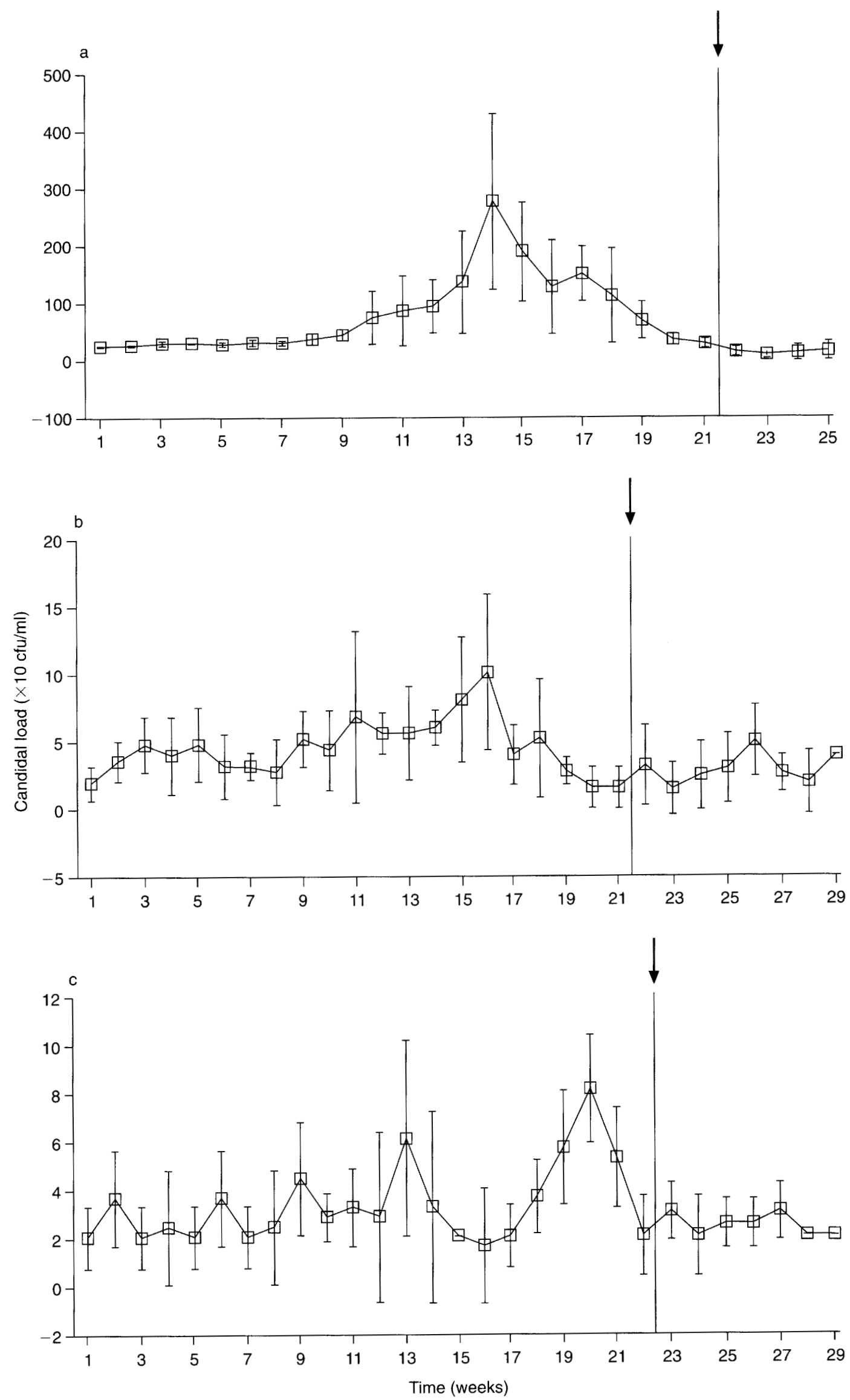

Fig. 1. The mean oral load of: a, C. albicans; b, C. krusei CK9; c, CK13 before and after $(\rightarrow)$ administration of cyclophosphamide in SD rats. (Note the scale difference in the $\mathrm{Y}$ axis.) 


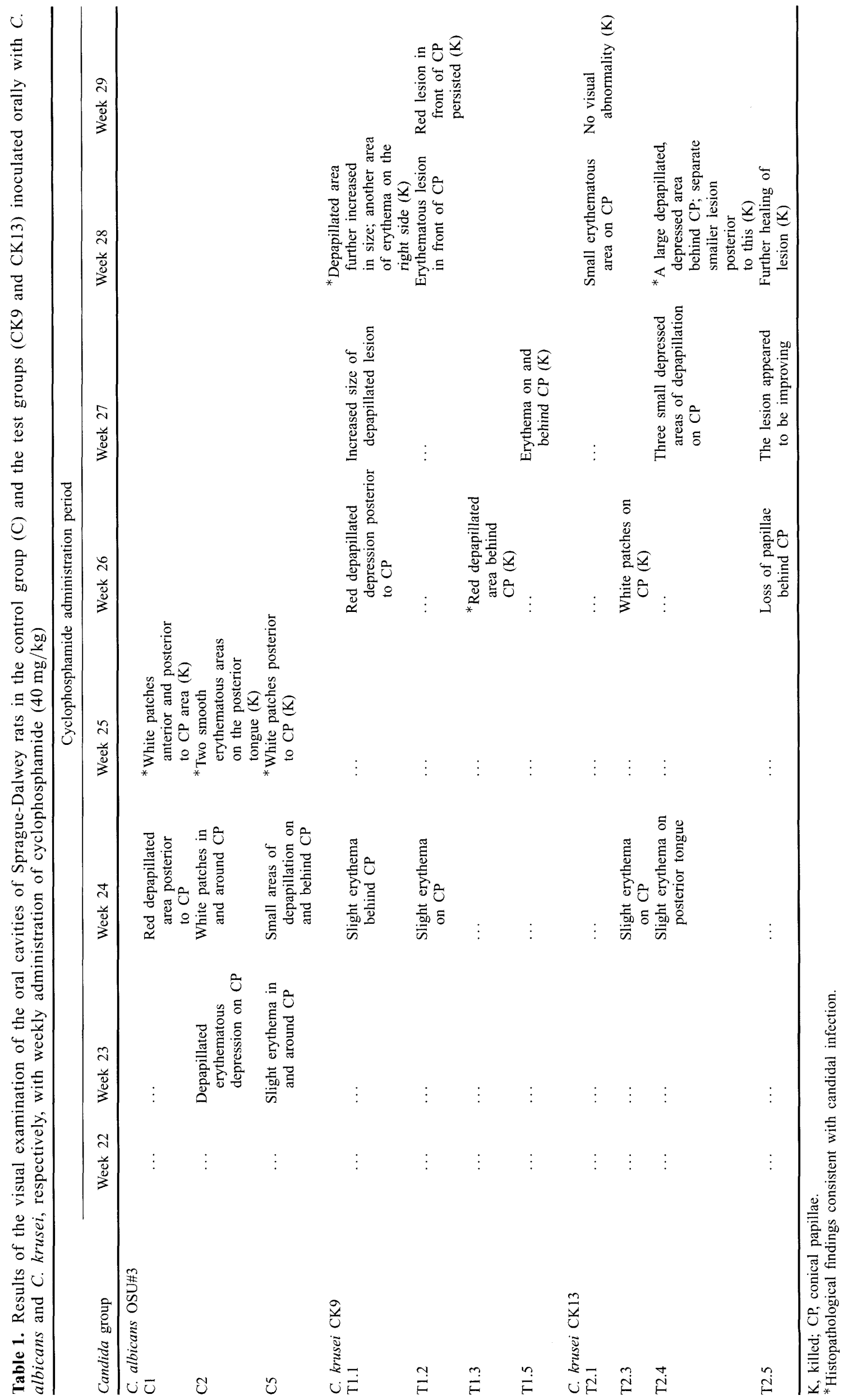




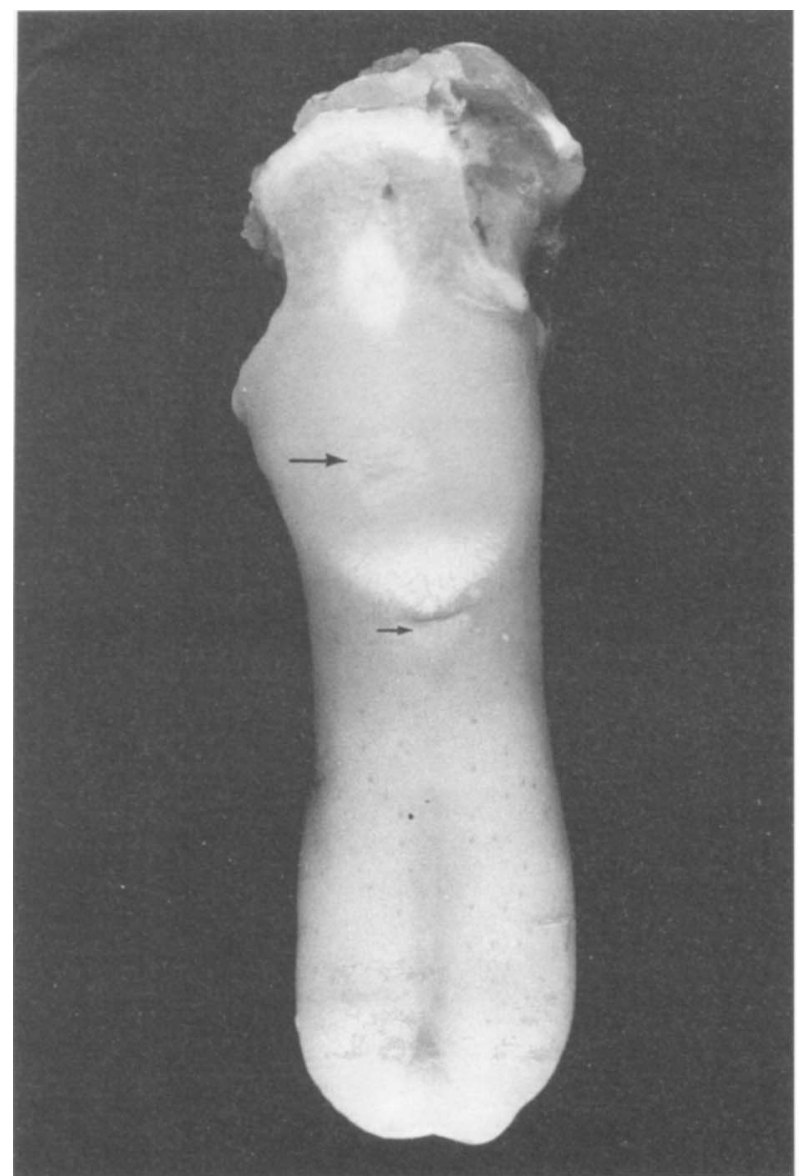

Fig. 2. Macroscopic appearance of Sprague-Dawley rat tongue $\mathrm{C} 1$, infected with $C$. albicans OSU\#3 after 25 weeks of sequential oral inoculation with the yeast and 4 weeks of cyclophosphamide administration. Note the areas of homogeneous leucoplakia $(\rightarrow)$ and speckled leucoplakia $(\longrightarrow)$.

lesions to be detected visually with $C$. krusei than with $C$. albicans. The time required for discernible changes on the tongue mucosa varied between 3 and 8 weeks and 3 and 7 weeks with C. krusei isolates CK9 and CK13, respectively, compared with 2-4 weeks for C. albicans (Table 1). A total of three of eight rats infected with $C$. krusei demonstrated candidal infection after cyclophosphamide treatment, compared with all three rats in the $C$. albicans group. The lingual mucosal lesions and their histopathological changes in both groups of rats infected with $C$. krusei CK9 and CK13 were essentially very similar to the lesions produced by $C$. albicans OSU\#3. PAS-stained sections showed hyphal infiltration of the cornified layers by $C$. krusei (Fig. 4b) which were generally shorter than those of $C$. albicans, and penetrated a relatively short depth of the keratinised epithelium varying between 3.73 and $5.07 \mu \mathrm{m}$ and 5.01-8.34 $\mu \mathrm{m}$ for strains CK9 and CK13, respectively compared with $5.00-17.00 \mu \mathrm{m}$ for $C$. albicans. However, the number of hyphae penetrating the oral epithelium was relatively fewer and more sparsely distributed for $C$. krusei than with C. albicans. C. albicans hyphae penetrating the oral epithelium appeared in clusters on the lingual epithelium, compared with isolated, individual hyphae in the case of $C$. krusei. Oral lesions were recorded for the other five rats, but no yeasts were demonstrated histologically. Any lesions observed appeared to recover during a short period (Table 1). For instance, after 3 weeks of inoculation with $C$. krusei CK9, rat T1.2 in test group 1 showed mild erythema on the conical papillae area, but during the following 3 weeks of the test period the mucosal surface returned to normal, although an ill-defined slightly erythematous area appeared in front of the conical papillae area during the eighth week.

\section{Discussion}

A world-wide increase in $C$. krusei infection in immunocompromised patient groups has been described recently [15]. In these individuals the clinical presentation of the disease is influenced not only by the compromised immune status, but also by other factors such as the presence of catheters due to intravenous hyperalimentation, oral prostheses, endocrine disturbances and use of antibiotics or steroids [21]. Despite the increased morbidity caused by $C$. krusei, the approaches used in the investigation of mucosal candidosis have focused mainly on the most virulent species, C. albicans, and to our knowledge there are no animal studies pertaining to oral $C$. krusei infection.

Several animal models such as monkeys, rabbits, rats and mice have been developed to study the pathogenesis of oral candidosis. The disadvantages of the monkey and the rabbit model are their cost, and inconsistent oral infection in different hands [22-25]. Hence a smaller, less expensive rat model developed by Jones and Adams [5] has proved to be the most popular for the study of mucosal candidosis $[6,9-12,19,26-$ 29]. Such animal studies have contributed significantly towards the understanding of the relationship between C. albicans and its host. Hence the Sprague-Dawley rat model was chosen for the current study, and has proved to be reliable for experimental oral infection with $C$. krusei, albeit under immunosuppression.

Tetracycline was used throughout the current study as it is known that a tetracycline-laced diet and repeated yeast inoculation are necessary to maintain prolonged oral colonisation and infection in Sprague-Dawley rats $[19,28-33]$. Although there is a single report indicating that tetracycline exposure is not an essential prerequisite for initiating rat oral lesions, the authors acknowledged that the drug increases the overall size of the lesions [10]. In addition to tetracycline, a diet high in carbohydrate further promotes the oral carriage of yeasts and their infectivity [19]. For these reasons, and also because of the well-known low pathogenicity of C. krusei, it was decided to administer a carbohydrate-rich diet plus tetracycline. Nonetheless, these measures together with repeated yeast inoculation 

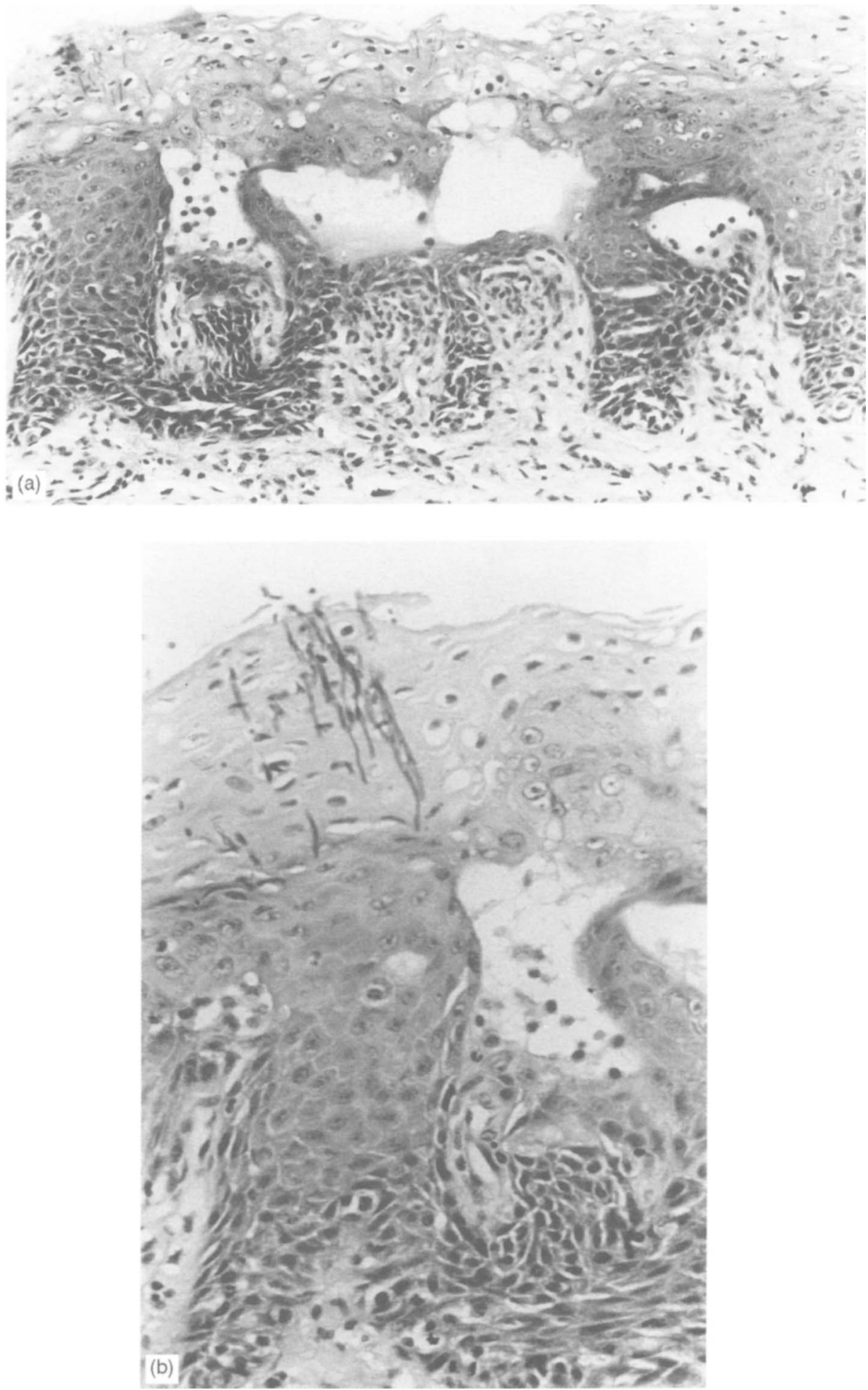

Fig. 3. (a) Histopathological section of leucoplakia on dorsal tongue of rat $\mathrm{C} 1$ infected with $C$. albicans OSU\#3. Note the loss of filiform papillae and vesicle formation and polymorph infiltration in the parakeratotic, oedematous epithelium $(\mathrm{H} \& \mathrm{E}, \times 200)$. (b) Section illustrating $C$. albicans hyphae infiltrating the cornified layers of the inflamed lingual epithelium (PAS, $\times 400)$.

failed to initiate oral lesions in immunocompetent rats, with either Candida species. This may be due to inbreeding of rats as $C$. albicans OSU\#3 is known to initiate infection under these experimental conditions.

Most studies that have employed animal models to mimic oral candidosis have recorded the carriage of candida as positive or negative by the culture of swabs. In this investigation, a semi-quantitative technique was used to assess oral yeast carriage, as described previously $[9,28]$. The consistent oral carriage of $C$. albicans OSU\#3 observed in this study 

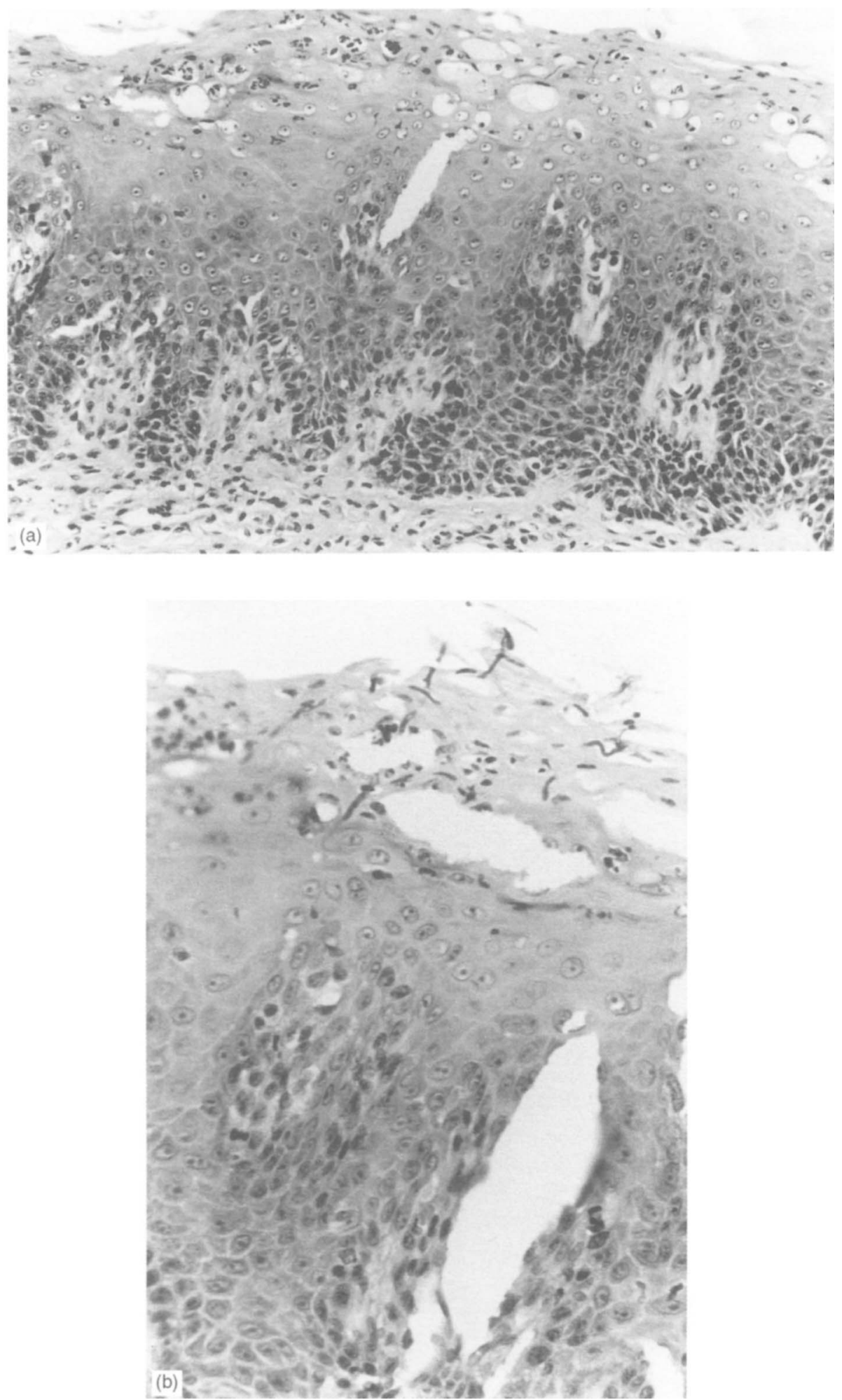

Fig. 4. (a) Histopathological section of a depapillated area on dorsal tongue of rat T1.1 infected with C. krusei CK9. Note the loss of papillae and orthokeratinisation. The infected superficial layers of the epithelium are oedematous with polymorph infiltration and vesicle formation $(\mathrm{PAS}, \times 200)$. (b) Periodic acid-Schiff stain to confirm epithelial invasion by C. krusei $($ CK9) hyphae $(\mathrm{PAS}, \times 400)$.

over 21 weeks was different to the sporadic yeast isolation reported by Allen et al. [28] who employed the identical yeast strain. This discrepancy may be due to the tetracycline-free, low carbohydrate diet employed by Allen et al. [28]. Dietary carbohydrates potentiate oral candidal carriage in a number of ways, including enhancement of yeast adhesion to mucosae [34], and production of metabolic carboxylic acids leading to a reduction in $\mathrm{pH}$ [35] with consequent activation of acidic proteases [36] and phospholipases 
[37]. Tetracycline, on the other hand, helps to suppress the commensal bacteria, thereby promoting yeast growth $[38,39]$.

It would appear that this is the first documented study on the oral carriage of $C$. krusei in an animal model. The results indicated that $C$. krusei isolates were able to colonise the rat tongue mucosa, but in smaller numbers than $C$. albicans, despite the use of equal inocula at an identical frequency and the same conditions. Thus, on occasions, some animals failed to yield $C$. krusei in swab cultures, whereas $C$. albicans was grown from all swab cultures throughout the experimental period of 29 weeks (Fig. 1). Furthermore, in terms of the numbers of organisms colonising the oral mucosa, the prevalence of C. albicans was much higher (c. 12-fold) than that of $C$. krusei, implying clearly that the colonisation potential is an intrinsic ability of each Candida spp., and may vary between isolates within a given species. Nonetheless, further studies with large numbers of isolates of each species need to be conducted to verify such inter-species attributes.

The lower oral carriage of $C$. krusei, in general, may reflect its reduced ability to adhere to buccal epithelial cells when compared with $C$. albicans. In-vitro studies have shown reduced adherence of $C$. krusei to buccal and vaginal epithelial cells in comparison with $C$. albicans, C. tropicalis, C. stellatoidea and C. parapsilosis [40]. In one study, an 11-fold increase in adherence of $C$. albicans to buccal epithelial cells compared with $C$. krusei was observed [41]. These observations broadly concur with our previous studies in which adhesion of $C$. krusei to buccal epithelial cells from healthy individuals was 10 times less than that of C. albicans [16].

Although $C$. albicans was isolated consistently from all five immunocompetent rats, no candidal infection was observed either on gross or histopathological examination of tongue tissues. In previous similar studies conducted by Russell and Jones [19] and Hassan et al. [32], candidal infection, indicated by mycelial penetration of the superficial lingual epithelium, was noted in $63 \%$ and $60 \%$ of rats, respectively. It is difficult to explain why the animals in the present study did not develop infection despite the fact that the $C$. albicans OSU\#3 strain used was capable of initiating oral lesions previously $[9,10]$. Perhaps the attenuated virulence of a subcultured isolate or the higher natural immunity of rats derived from a local stock may account for this observation.

Under cyclophosphamide-mediated immunosuppressed conditions, $C$. albicans initiated oral lesions in all three rats. Lingual infection confirmed by histopathological observation resembled features described by previous investigators $[19,32]$. However, large vesicles with polymorph infiltration were also observed in the stratum spinosum (Fig. 4a), a feature not previously reported. This may be due to candidal infection superimposed on cyclophosphamide-mediated changes in a rapidly proliferating epithelial cell compartment [42].

The inability of $C$. krusei to infect healthy animals was not surprising, as this opportunist pathogen is isolated mainly from patients with reduced defences [43-45]. However, both C. krusei isolates were able to induce lingual infection to some extent in animals that were rendered immunocompromised. Hyphal penetration of the stratum corneum of the lingual mucosa was evident, resembling the histopathological changes induced by $C$. albicans. However, the intra-epithelial vesicles and hyphal penetration appeared to a lesser extent in $C$. krusei lesions. Examination of the erythematous lingual areas of the five rats in test groups 1 and 2 that did not show evidence of candidal infection revealed only mild cellular disorganisation in the basal and parabasal cell layers with occasional lymphocytic infiltration. These observations have shown that oral candidosis can be induced, albeit with some difficulty, in the rat model by the repeated oral inoculation of immunosuppressed rats with $C$. krusei. The fact that only c. 33\% of compromised animals developed the infection substantiates the lesser virulence potential of $C$. krusei compared with $C$. albicans.

To conclude, these results confirm that $C$. krusei is an opportunist pathogen that may cause disease when host defences are impaired, and concur with clinical epidemiological data which designate the organism as an emerging pathogen, especially in compromised hosts [15]. Furthermore, the Sprague-Dawley rat model appears to be useful for future workers to simulate in vivo the pathogenicity of Candida spp. with low virulence, such as $C$. parapsilosis, $C$. glabrata, etc., under immunocompromised situations.

This research was supported by the Committee for Research and Conference Grants of the University of Hong Kong (grant nos. $335 / 268 / 0004$ and $335 / 268 / 0006$ )

\section{References}

1. Cawson RA, Lehner T. Chronic hyperplastic candidiasis candidal leukoplakia. $B r J$ Dermatol 1968; 80: 9-16.

2. Cawson RA. Chronic oral candidosis, denture stomatitis and chronic hyperplastic candidosis. In: Winner HI, Hurley R (eds) Symposium on Candida infections. Edinburgh, Livingstone. 1966: $138-153$

3. Howlett JA. The infection of rat tongue mucosa in vitro with five species of Candida. J Med Microbiol 1976; 9: 309--316.

4. Partridge BM. The use of the chorioallantoic membrane of the developing chick for culture of dermatophytes: a modified technic; a preliminary report upon its use for serial passage. $J$ Invest Dermatol 1959; 32: 605-619.

5. Jones JH, Adams D. Experimentally induced acute oral candidosis in the rat. $\mathrm{Br} J$ Dermatol $1970 ; 83: 670-673$.

6. Adams D, Jones JH. Life history of experimentally induced acute oral candidiasis in the rat. $J$ Dent Res 1971; 50: 643-644.

7. Jones, JH, Russell C. The histology of chronic candidal 
infection of the rat's tongue and its relevance to human oral leukoplakia. J Clin Pathol 1973; 26: 390.

8. Jones JH, Russell C. The time scale of penetration of the rat's oral epithelium by $C$. albicans. International Research Communications System 1973; 73-12, 6-3-5.

9. Allen CM, Beck FM. Strain-related differences in pathogenicity of Candida albicans for oral mucosa. J Infect Dis 1983; 147: 1036-1040.

10. Allen CM, Beck FM, Lurie FA, Pinsky HM. Role of tetracycline in pathogenesis of chronic candidiasis of rat tongues. Infect Immun 1985; 47: 480-483.

11. Allen CM, Beck FM. Differences in mucosal reaction related to Candida albicans isolates. J Oral Pathol 1987; 16: 89-93.

12. Allen CM. Animal models of oral candidiasis: a review. Oral Surg Oral Med Oral Pathol 1994; 78: 216-221.

13. Horn R, Wong B, Kiehn TE, Armstrong D. Fungemia in a cancer hospital: changing frequency, earlier onset, and results of therapy. Rev Infect Dis 1985; 7: 646-655.

14. Wingard JR, Merz WG, Rinaldi MG, Johnson TR, Karp JE, Saral R. Increase in Candida krusei infection among patients with bone marrow transplantation and neutropenia treated prophylactically with fluconazole. $N$ Engl J Med 1991; 325: 1274-1277.

15. Samaranayake YH, Samaranayake LP. Candida krusei: biology, epidemiology, pathogenicity and clinical manifestations of an emerging pathogen. J Med Microbiol 1994; 41: 295-310.

16. Samaranayake YH, Wu PC, Samaranayake LP, So M, Yuen KY. Adhesion and colonisation of Candida krusei on host surfaces. $J$ Med Microbiol 1994; 41: 250-258.

17. Samaranayake YH, Wu PC, Samaranayake LP, So M. Relationship between the cell surface hydrophobicity and adherence of Candida krusei and Candida albicans to epithelial and denture acrylic surfaces. APMIS 1995; 103: 707-713.

18. Silverman S, Migliorati CA, Epstein JB, Samaranayake LP. Laboratory diagnosis of oral candidosis. In: Samaranayake LP, MacFarlane TW (eds) Oral candidosis. London, Wright. 1990: 213-237.

19. Russell C, Jones JH. Effects of oral inoculation of Candida albicans in tetracycline-treated rats. $J$ Med Microbiol 1973; 6: 275-279.

20. Darcie JV, Lewis SM. Basic haematological techniques, In: Practical haematology, 7 th edn. London, Churchill Livingstone. 1991.

21. Odds FC. Candida and candidosis, 2nd edn. London, Bailliere Tindall. 1988.

22. Budtz-Jorgensen E. Denture stomatitis IV. An experimental model in monkeys. Acta Odontol Scand 1971; 29: 513-526.

23. Budtz-Jorgensen E. Cellular immunity in acquired candidiasis of the palate. Scand J Dent Res 1973; 81: 372-382.

24. Olsen I, Haanaes HR. Experimental palatal candidosis and saliva-flow in monkeys. Scand J Dent Res 1977; 85: 135-141.

25. Mackinnon JE. Nuevo sentido de variacion en Mycotorula (monilia) albicans. Archivos Sociedad de Biologia de Montevideo 1936; 7: 162-163.

26. Russell C, Jones JH. The effects of oral inoculation of the yeast and mycelial phases of Candida albicans in rats fed on normal and carbohydrate rich diets. Arch Oral Biol 1973; 18: $409-412$.

27. Russell C, Jones JH, Gibbs ACC. The carriage of Candida albicans in the mouths of rats treated with tetracycline briefly or for a prolonged period. Mycopathologia 1976; 58: 125-129.

28. Allen CM, Blozis GG, Rosen S, Bright JS. Chronic candidiasis of the rat tongue: a possible model for human median rhomboid glossitis. J Dent Res 1982; 61: 1287-1291.

29. Fisker AV, Rindom Schiott C, Philipsen HP. Long-term oral candidosis in rats. APMIS 1982; 90: 221-227.

30. Jones JH, Russell C, Young C, Owen D. Tetracycline and the colonization and infection of the mouths of germ-free and conventionalized rats with Candida albicans. J Antimicrob Chemother 1976; 2: 247-253.

31. Russell $\mathrm{JH}$, Jones $\mathrm{JH}$. The histology of prolonged candidal infection of the rat's tongue. $J$ Oral Pathol 1975; 4: 330-339.

32. Hassan OE, Jones JH, Russell C. Experimental oral candidal infection and carriage of oral bacteria in rats subjected to a carbohydrate-rich diet and tetracycline treatment. $J$ Med Microbiol 1985; 20: 291-298.

33. Fisker AV, Rindom Schiott C, Philipsen HP. Short-term oral candidosis in rats, with special reference to the site of infection. APMIS 1982; 90: 49-57.

34. Samaranayake LP, MacFarlane TW. On the role of dietary carbohydrates in the pathogenesis of oral candidosis. FEMS Microbiol Lett 1985; 27: 1-5.

35. Samaranayake LP, Hughes A, Weetman DA, MacFarlane TW Growth and acid production of Candida species in human saliva supplemented with glucose. J Oral Pathol 1986; 15: $251-254$.

36. Samaranayake LP, Hughes A, MacFarlane TW. The proteolytic potential of Candida albicans in human saliva supplemented with glucose. J Med Microbiol 1984; 17: 13-22.

37. Samaranayake LP, Raeside J, MacFarlane TW. Factors affecting the phospholipase activity of Candida species in vitro. Sabouraudia 1984; 22: 201-207.

38. Seelig MS. Mechanisms by which antibiotics increase the incidence and severity of candidiasis and alter the immunological defenses. Bacteriol Rev 1966; 30: 442-459.

39. Solomon P. Oral moniliasis complicating combined broadspectrum antibiotic and antifungal therapy. $N$ Engl $J \mathrm{Med}$ 1961; 265: 847-848.

40. King RD, Lee JC, Morris AL. Adherence of Candida albicans and other Candida species to mucosal epithelial cells. Infect Immun 1980; 27: 667-674.

41. Tobgi RS. Differences in the pathogenic potential of Candida species especially $C$. albicans. $\mathrm{PhD}$ thesis, University of Glasgow, 1989.

42. Ferguson MM. Oral mucous membrane markers of internal disease; part II: disorders of the endocrine system, haemopoietic system and nutrition. In: Dolby AD (ed) Oral mucosa in health and disease, Oxford; Blackwell. 1975: 249-250.

43. Merz WG, Karp JE, Schron D, Saral R. Increased incidence of fungemia caused by Candida krusei. J Clin Microbiol 1986; 24: $581-584$.

44. Nguyen VQ, Penn RL. Candida krusei infectious arthritis. A rare complication of neutropenia. $A m J$ Med 1987; 83: 963-965.

45. McQuillen DP, Zingman BS, Meunier F, Levitz SM. Invasive infections due to Candida krusei: report of ten cases of fungemia that include three cases of endophthalmitis. Clin Infect Dis 1992; 14: 472-478. 